\title{
COMPARATIVE STUDY OF JUSTIFICATION METHODS IN RECOMMENDER SYSTEMS: EXAMPLE OF INFORMATION ACCESS ASSISTANCE SERVICE (IAAS)
}

\author{
Kyelem Yacouba ${ }^{1}$, Kabore Kiswendsida Kisito $^{1}$, \\ Ouedraogo Tounwendyam Frédéric ${ }^{2}$ and Sèdes Florence ${ }^{3}$ \\ ${ }^{1}$ Department of Informatic, Université Joseph Ki-Zerbo, \\ Ouagadougou, Burkina Faso \\ ${ }^{2}$ Department of Informatic, Université Norbert Zongo, \\ Koudougou, Burkina Faso \\ ${ }^{3}$ IRIT, Toulouse, France
}

\begin{abstract}
Justification of recommendations increases trust between users and the system but also generates more relevant recommendations than recommendation systems that do not incorporate it. That is why, we conducted a justification study of the recommendation for IAAS. Our comparative study shows that IAAS, which currently does not offer the opportunity to justify recommendations, needs to be improved. From the analysis of justification methods studied in this work, it appears that none of these methods can be used effectively in IAAS. That is why, we proposed a new IAAS architecture that deals separately with item classification and the extraction of the justification has added the item during recommendation generation. The item selection method remains unchanged as we plan to implement a new strategy to filter user's reviews should now be extended to four elements: the documentary unit, the group of users, the justification and the weight. Opinion $A=(U D, G, J, a)$. Where UD represents the documentary unit, $G$ the user group, $J$ is the justification and $a$ is the weight of the recommendation.
\end{abstract}

\section{KEYWORDS}

IAAS, Justification in Recommender Systems, users reviews, weight of reviews.

\section{INTRODUCTION}

In order to facilitate the access to the information contained in information systems, recommendation systems have been developed; these systems use the actions of users realized on the system to filter information. These recommendation systems have undergone a high evolution and have allowed the implementation of several approaches such as: the content-based filtering approach, the collaborative filtering approach, the hybrid approach, the demographic approach and the social approach etc [19]. All these approaches have been proposed in order to produce relevant recommendations to users. As for the collaborative filtering approach, the system uses the ratings of similar users to provide them recommendations [19]. With this approach, several algorithms allowing to provide more accurate recommendations to the user have been developed, for instance IAAS. 
IAAS, Information Access Assistance Service is a collaborative filtering recommendation system. This system aims to be applied in several domains such as videos, audios, images and documents. It uses the notion of voting as a technique for evaluating items. This vote is carried out by the user after having taken note of the document. This user estimates that the document is important for one or more other users, and it materializes it through an opinion $[2,3,4][20]$.

All recommendation approaches have produced algorithms to provide more accurate recommendations to the user. However, the accuracy of the recommendation and its acceptance improves when the user is able to understand the limitations and benefits of the recommendation. Otherwise, the user must receive the recommendation with the reasoning behind it [5],[18]. Thanks to these observations and to the evolution of recommendation systems which is to improve the interface through the justification of recommendations, the notion of justification has been introduced in recommendation systems. There are several types of justifications: keyword justification, influence based justification, content based justification, users reviews justification and comparative justification etc [1],[5],[7]. The justified recommendation gives credibility to the recommendation system.

As for IAAS, which does not take into account the notion of justification of recommended items must be thought of in order to improve the relevance of the recommendation. So, we will see through this study how to justify the recommendations with the IAAS algorithm. To achieve this, we will review the literature on the justification methods used in the recommendation systems in order to compare these methods. Then we position ourselves in relation to IAAS. To do this, we will first present our literature review on IAAS and the justification methods. Then, we will draw up the comparative table and we will finish by summarizing.

\section{RELATED WORK}

\subsection{Information Access Assistance Service (IAAS)}

In the IAAS recommendation system, the users appreciate the different documentary units during their consultation and this appreciation is carried out by giving a grade to the documentary unit. Thus a user gives his opinion (A) which is a grade 'a' ranging from 1 to 10 , on a documentary unit (UD) and a given group (G). Hence for [2], [3], the opinion is defined by the following triplet A $=(\mathrm{UD}, \mathrm{G}, \mathrm{a})$.

The system collects all these relevance notices and proceeds implicitly to the calculation and ranking of the relevant items for the user. As each documentary unit can receive several relevance notices, the notion of recommendation weight $\mathrm{Pk}(\mathrm{UDiGj})$ has been proposed by [2],[3],[4]. The calculation allows to give a weight to each item to be recommended. As a group of users can receive the recommendation of the same document through several users, the total weight is calculated from the following formula [2],[3],[4]:

$\operatorname{Pk}\left(U D_{i} G_{j}\right)=\operatorname{sum}\left(U D_{i}, G_{j}, a\right)(1)$

In the case of a document that has no relevance notice by a user its recommendation weight pk is zero [2],[3],[4].

$P k\left(U D_{i} G_{j}\right)=0 \quad(2)$

In IAAS, a user's connection to the system is analyzed as a request to transmit recommendations. The recommendation transmitted to a user contains all the documents recommended to his group. 
For each user group, after calculating the recommendation weight for each item, IAAS orders the list of documents based on the relevance value. The relevance value is expressed by the following formula [2]:

Relevance $P_{i, j}=\ln \left(1+P k\left(U D_{i} G_{j}\right)\right)$

The documents are ordered according to the decreasing values of $\mathrm{P}_{\mathrm{i}, \mathrm{j}}$ and then reordered several times according to the users' profile to be personalized to each user of the group.

The notion of profile is very fundamental in IAAS. Indeed, in order to personalize the recommendations to the users, [2],[3],[4] have implemented the user profile and the document profile. These profiles are schemas that can be consulted in the works of [2], [3], [20].

\subsection{Justification approaches}

We present in this part of our work, the summary of the work already done on the approaches of justification in the systems of recommendations.

\subsubsection{Approach Feature-Weighted Nearest Biclusters (FWNB)}

The FWNB approach is built around four elements that are user group creation, keyword weighting, neighborhood formation, and recommendation and justification generation [5].

- Creation of user groups: it is based on the formation of user and item biclusters. The formation of these biclusters is done thanks to the similarity between users and items they have already evaluated. This bicluster formation is done automatically using the xMotif algorithm [6].

- Weight of keywords: The objective of [5] in constructing keyword weights is to find the distinct keywords that best describe the users' preferences. For this purpose, [5] used the similarity matrix between keywords and users. The weight of keyword f for a user $u$ is calculated as follows:

$$
\begin{gathered}
W(u, f)=\mathrm{FF}(\mathrm{u}, \mathrm{f}) * \operatorname{IUF}(\mathrm{f}) \\
\operatorname{IUF}(f)=\log \frac{\| \mathrm{U}]}{\mathrm{UF}_{(\mathrm{f})}}
\end{gathered}
$$

$|\mathrm{U}|$ : total number of users and $\mathrm{UF}(\mathrm{f})$ : number of users in which the keyword $\mathrm{f}$ appears at least once.

$\mathrm{FF}(\mathrm{u}, \mathrm{f})=\mathrm{P}(\mathrm{u}, \mathrm{f})$ is the correlation between the user and the keyword $\mathrm{f}$.

Using the keyword user correlation matrix R_B (u,f), they generate the keyword weight matrix W_B from the formula W(u,f) [5].

- Neighborhood formation: This is the identification phase of the items and keywords to be recommended. All the items contained in the biclusters are candidates for recommendation as well as the keywords. Thus we determine the item and the justification for each user through the calculation of similarity between the user and his bicluster:

$\operatorname{sim}(u, b)=(1-a) \cdot \operatorname{sim}(u, b)+a \operatorname{sim}_{F}(u, b)$ 
$\operatorname{simI}(u, b)$ : similarity between the user and his item; $a \in[0,1]$; $\operatorname{simF}(u, b)$ : similarity between the user and keyword

- Recommendation and justification generation: the generation of the item to be recommended as well as the corresponding keyword is done by simultaneous identification of the items in the neighborhood of the bicluster of a user $u$ who :

$\checkmark$ are all preferred by other users according to the scores of the R_B matrix

$\checkmark$ contain the significant keywords of the matrix W_B.

The generated recommendation is the form, 'item $\mathrm{X}$ is recommended to you because it contains the keyword $\mathrm{f}$ that you have already evaluated in item $\mathrm{Y}$ '.

\subsubsection{Approach of Cataldo et al}

[7] was interested in building an effective justification designed on the basis of the distinctive and relevant terms for the item starting from the users' reviews. For him, the effective justification must include the relevant and distinctive terms of the items that are discussed in the reviews. The approach of [7] is structured as follows: terms extraction, terms ranking, sentences filtering and the text summarization.

- Feature extraction: The first step is to identify the features that deserve to be included in the final justification. Thus the strategy of [7] takes as input a set of reviews $R=\{r 1, r 2$. ..rn\} and produces a set of 4 -tuples ((ri, aij, rel(aij, ri), sent(aij, ri)). To extract the terms of the critics, [7] used the Kullback-Leibler divergence [8], which is a nonsymmetric measure of the difference between two distributions to construct an algorithm.

- Ranking the extracted terms: [7] proceeded to calculate the score of the extracted terms by the following formula:

$$
\operatorname{score}(a j)=\frac{\sum_{i=1}^{N} n_{a j, r i} * \operatorname{rel}\left(a_{j}, r_{i}\right) * \operatorname{sent}\left(a_{j}, r_{i}\right)}{\mathrm{N}}
$$

At the end, the terms are ranked in descending order and the K-first ones are labeled as main terms.

- Sentence filtering: After the identification of the terms, we proceed to a sentence filtering with the objective of filtering out the sentences that are considered not necessary in the final justification. To do this, we divide the criticisms ri $\in \mathrm{R}$ into sentences si1 .... sim. Then we check if the sentences respect in particular the criteria of content of the extracted terms. A top k of sentences are selected.

- Text summarization: the summary highlights the main contents of the item's reviews and maximizes both the coverage and the diversity of the justification while avoiding redundancy. The approach in [7] combines centroid-based text summarization [9], which has the advantage of being unsupervised, with a pre-trained neural language model, such as Word2Vec [10].

\subsubsection{Approach of Jianmo et al}

The generation of justifications for recommendations in [11] is done using a pipeline to identify candidate terms for justification and to form the users and item profiles from a corpus of reviews. The candidate terms are the reviews that the user had previously written. [11] constructs a dataset containing the custom's reviews for each user. The construction of the pipeline starts with the 
annotation of the reviews, then the classification of the annotated terms using the calculation of the distance between the selected terms and ends with the extraction of the justifications and the construction of the user and item profiles. Thus, for each user $u$ we build the pipeline using the reference justifications $\mathrm{D}=(\mathrm{fd} 1 ; \ldots ; \mathrm{dlr})$ consisting only of the justifications that the user had written.

Then we have the user profile composed of $\mathrm{A}=(\mathrm{a} 1 ; \ldots ; \mathrm{aK})$, we carry the most relevant ones and in the same way we build the items profile. For a user $\mathrm{u}$ and an item $\mathrm{i}$ as well as their reference justifications $\mathrm{Du}$ and $\mathrm{Di}$, and the user profile $\mathrm{Au}$ and $\mathrm{Ai}$ of the item; we predict the justifications $\mathrm{Ju} ; \mathrm{i}=(\mathrm{w} 1 ; \mathrm{w} 2 ; \ldots ; \mathrm{wt})$ that explain why the item $\mathrm{i}$ is important for the user u. [11] identifies the terms or phrases using [13] and linguistic analysis. After identification, [11] uses the BERT [14] method for automatic classification of justifications and uses Fine-grained Aspect Extraction [15] for the extraction and the profile construction. The approach of [11] uses two models in its approach which are: Reference-based Seq2Seq Model and Aspect Conditional Masked Language Model. The experimentation has shown that the former produces high quality justifications and the latter produces diverse justifications.

\subsubsection{Approach of Arpit et al}

The explanation-based recommendation is a new approach that unifies recommendation and explanation. The recommendation is modeled as a path finding problem in the item-item similarity graph [12].

Once a chain has been constructed for each candidate item, the top-n chains are iteratively selected based on their total coverage of the candidate item terms and their dissimilarities with other top-n chains. The approach of [12] is built on the generation of explanation chains and the evaluation of this chain.

Generation of explanation chains:

$\operatorname{rwd}(j, i, C)=\frac{\|\left(f_{j} \backslash \text { covered }(\mathrm{i}, \mathrm{C})\right) \cap f_{i} \|}{\left\|f_{i}\right\|}+\frac{\|\left(f_{j} \backslash \text { covered }(\mathrm{i}, \mathrm{C})\right) \cap f_{i} \|}{\left|f_{j}\right|}$

Evaluation of explanation chains:

$\operatorname{score}\left(\{C, i\}, C^{*}\right)=\frac{\sum_{j \in C} \operatorname{rwd}(j, i, C)}{\|\mathrm{C}\|+1}+\frac{\left\|\mathrm{C} \backslash \mathrm{u}_{j r \in C \cdot J^{\prime}}\right\|}{\| \mathrm{c} \mid+1}$

Then comes the selection of items to be presented to the user. The technique of [12] does not compute separately the selection of items and justifications.

\subsubsection{Approach of Or Biran et al}

[16] proposes an automatic prediction method using machine learning to produce simple, short, quality natural language justifications; through the use of application domain critics [8]. This approach has a message prediction structure and architecture.

[16] uses the Semantic Template Typed (STT) message structure which is a small semantic network of typed entity slots and relationships for prediction. A set of STTs have been created for each justification domain and use specific STTs to a domain, as well as template sets, from text corpora for words extraction. 
The template construction architecture of [16] consists of term selection and characterization, computation of certain quantities if any, and justification planning.

\section{ARCHITECTURE OF RECOMMENDATION JUSTIFICATION IN IAAS}

The figure 1 below expresses the idea of how the recommendation justification in IAAS that we want to implement works. The diagram shows three main entities which are the users, the workstation and the Information Access Assistance Service.

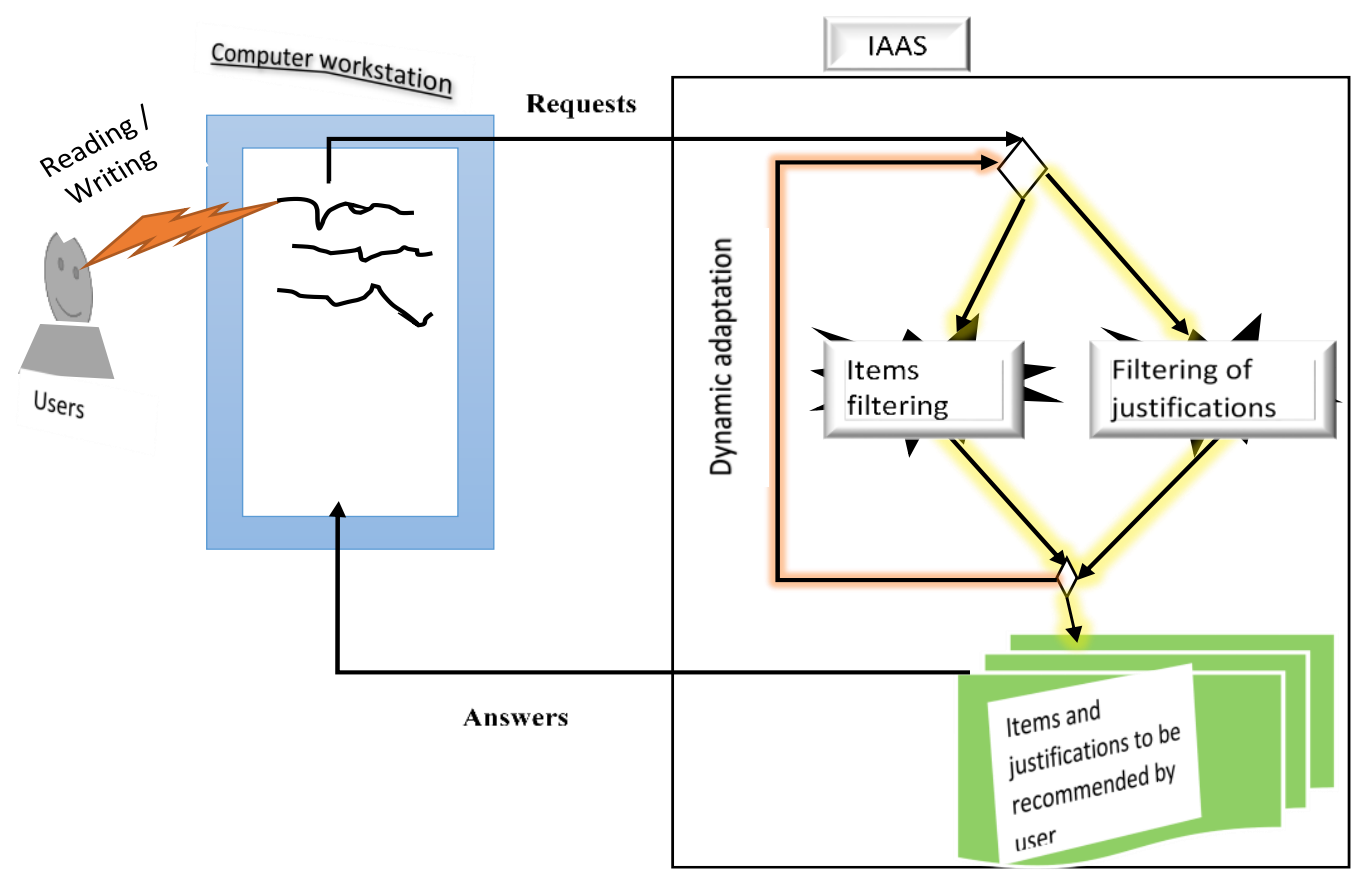

Figure. 1. Description of the recommendation justification in IAAS

Users give their opinions on the documents and at the same time note the justification of its opini on which is sent to IAAS for processing. In IAAS, we have separated the processing of the filtering of the documentary units and the justifications because the justifications are only texts that we want to process whereas the mechanism of filtering of the documents already developed in [2] is a manipulation of the numbers. After the separate processing of document and credential selections we collect them and then customize them to each user as [2] provides. Once a user logs in, the system returns his recommendations and it is still possible for him to make a new recommendation. In case a user recommends the same document to the same user group, the system uses the ranking already obtained to make a new readapatation of recommendation. This means that the recommendation is dynamic.

\section{Synthesis OF JUSTIFICATION APPROACHES IN RECOMMENDER SYSTEMS}

The table below summarizes the work already done in the area of justifications in recommender systems. The automatic summarization method column was realized after consulting [17]. 


\subsection{Summary table}

Table 1. Comparison of Some Justification Approaches in Recommender Systems

\begin{tabular}{|c|c|c|c|c|}
\hline Justifying & Methods & $\begin{array}{l}\text { Justification } \\
\text { filtering } \\
\text { mechanism }\end{array}$ & $\begin{array}{|ll|}\text { Automatic } & \text { text } \\
\text { summarization } & \\
\text { methods } & \\
\end{array}$ & $\begin{array}{l}\text { Justification } \\
\text { style }\end{array}$ \\
\hline $\begin{array}{l}\text { User- } \\
\text { evaluated } \\
\text { data } \\
{[5]}\end{array}$ & $\begin{array}{l}\text { Creation of } \\
\text { groups } \\
\text { Weight of } \\
\text { terms } \\
\text { Formation of } \\
\text { neighbors [5] }\end{array}$ & $\begin{array}{l}\text { Features weight } \\
\text { Features } \\
\text { frequency } \\
\text { [5] }\end{array}$ & $\begin{array}{l}\text { Digital approach: } \\
\text { Learning-based methods }\end{array}$ & $\begin{array}{l}\text { Keywords } \\
\text { and influence } \\
\text { [5] }\end{array}$ \\
\hline $\begin{array}{l}\text { Users reviews } \\
{[7]}\end{array}$ & $\begin{array}{l}\text { Words } \\
\text { extraction } \\
\text { Words } \\
\quad \text { classificati } \\
\text { on } \\
\text { Sentence } \\
\text { filtering } \\
\text { Text } \\
\text { summariza } \\
\text { tion } \\
\text { [7] }\end{array}$ & $\begin{array}{l}\text { Kl-divergence } \\
\text { Term score } \\
\text { Term ranking } \\
\text { Sentences } \\
\text { extraction [7] }\end{array}$ & $\begin{array}{l}\text { Digital approach: Methods } \\
\text { based on statistical } \\
\text { calculations }\end{array}$ & $\begin{array}{l}\text { Summary } \\
\text { reviews } \\
{[7]}\end{array}$ \\
\hline $\begin{array}{l}\text { Key words } \\
\text { written on } \\
\text { the items } \\
{[11]}\end{array}$ & $\begin{array}{l}\text { Extration of } \\
\text { explanation } \\
\text { chains } \\
\text { Evaluation of } \\
\text { explanatio } \\
\text { n chains } \\
{[11]} \\
\end{array}$ & $\begin{array}{l}\text { Construction of } \\
\text { item chains } \\
{[11]}\end{array}$ & ----- & $\begin{array}{l}\text { Keywords } \\
{[11]}\end{array}$ \\
\hline $\begin{array}{l}\text { User- } \\
\text { evaluated } \\
\text { data [12] }\end{array}$ & \begin{tabular}{|l} 
Pipeline \\
Dataset [12]
\end{tabular} & $\begin{array}{l}\text { Annotation, } \\
\text { classification } \\
\text { and extraction of } \\
\text { terms [12] }\end{array}$ & $\begin{array}{l}\text { Digital approach: } \\
\text { Learning-based methods }\end{array}$ & $\begin{array}{l}\text { Summary of } \\
\text { reviews [12] }\end{array}$ \\
\hline $\begin{array}{l}\text { Corpus of } \\
\text { texts [16] }\end{array}$ & $\begin{array}{l}\text { Selection and } \\
\text { characteriz } \\
\text { ation of } \\
\text { terms } \\
\text { Planning of } \\
\text { the } \\
\text { justification } \\
\text { [16] }\end{array}$ & $\begin{array}{l}\text { STT : it is used } \\
\text { to predict the } \\
\text { message }[16]\end{array}$ & \begin{tabular}{|l|} 
Symbolic approach: \\
Learning-based methods
\end{tabular} & \begin{tabular}{|l|} 
Content and \\
influence \\
{$[16]$}
\end{tabular} \\
\hline
\end{tabular}

\subsection{Positioning for justification in IAAS}

Our analysis is conducted based on the IAAS literature review, the definition of recommendation justification in IAAS and the summary table of justification approaches. As in IAAS users give their opinions on the documents so they must provide their reviews at the same time. Also the fundamental concept in IAAS is that the user gives a weight to each item that is used to manage and filter documents. So it is better that we use these same weights to add to the user reviews that will be used as justification filtering strategy. As filtering mechanisms for existing evidence from 
the table, only [5] uses the weight of reviews and of the frequencies to filter the justification but does not use user reviews as justification. On the other hand, in the case of the approaches studied [16], it applies only in expert system, whereas IAAS is not an expert system, so this strategy does not interest us in this work. [11] cannot be used because the keywords written on the items are used to filter and order the list of recommendations to justify. The approaches of [5] and [12] use the evidence already assessed by users to automatically generate new justified recommendations using the numerical approach and learning based methods as a text summary tool.

The approach of [7] seems to have a very similarity because for [7], the reviews are entered by the users and the system collects all the reviews and then proceeds to process it separately and personalizes the recommendations. Only that in [7] the reviews are not accompanied by weight. We also have the summary method which is focused on statistical calculations which will be used in the case of IAAS since our reviews will carry weights. The test domain of [7] is different from that of IAAS because [7] is used in the domain of cinema while IAAS is used on the documents, videos, audios and images.

Therefore, of all the approaches that we studied, there is no case for giving weight to reviews. Based on this comparison, we are going to set up a new approach of recommendation justification which will take into account the opinions of users. Instead of a notice being a triplet as proposed by [2], it must be a quadruplet to take the justifications written by the users. Thus for the justification in IAAS a notice is now a quadruplet noted $A=(U D, G, J, a)$ where $J$ represents the justification. This message is sent to IAAS for processing as shown in figure 1.

This study does not question what is done on IAAS but aims to improve it by adding the justification. We will work on keeping the item selection technique and similarly we will develop a module for the processing of justifications.

\section{Conclusions}

In this paper, a literature review has been conducted on the different approaches to justification of recommendations and the Information Access Assistance Service. Then, a comparative study of these approaches allowed us to position ourselves on the justification approach in IAAS. Our contribution lies in the fact that at the end of our study we propose that the notices in IAAS should be a quadruplet instead of a triplet [2]. It also appears from our study that no approach can be used properly with IAAS, hence our immediate perspective to propose a specific justification approach to IAAS and then implement this approach.

\section{REFERENCES}

[1] Cataldo M., Alain D.S., Christoph T., Amon R., and Giovanni S. 2021. Exploring the Effects of Natural Language Justifications in Food Recommender Systems. In Proceedings of the 29th ACM Conference on User Modeling, Adaptation and Personalization (UMAP '21), June 21-25, 2021, Utrecht, Netherlands. ACM, New York, NY, USA, 16 pages. https://doi.org/10.1145/3450613.3456827

[2] Kabore, K. , Peninou, A., Sié, O. , Sèdes, F. Implementing The Information Access Assistant Service (IAAS) For An Evaluation. Int. J. Internet Technology and Secured Transactions, Vol. 6, No. 1, 2015 (2015)

[3] Kabore, K. , Sié, O. , Sèdes, F. Information Access Assistant Service (IAAS). In The 8th International Conference for Internet Technology and Secured Transactions (ICITST-2013), IEEE UK/RI Computer Chapter, London, UK, December 9-12, (2013).

[4] Kiswendsida Kisito Kaboré : Système d'aide pour l'accès non supervisé aux unités documentaire. Thèse de doctorat du l'Université de Ouaga 1 Pr Joseph KI-ZERBO, Janvier 2018. 
[5] Panagiotis S., Alexandros N., and Yannis M. Providing Justifications in Recommender Systems. IEEE TRANSACTIONS ON SYSTEMS, MAN, AND CYBERNETICS-PART A: SYSTEMS AND HUMANS, VOL. 38, NO. 6, NOVEMBER 2008 pp. 1262-1272

https://ieeexplore.ieee.org/abstract/document/4648950

[6] T. Murali and S. Kasif, "Extracting conserved gene expression motifs from gene expression data," in Proc. Pacific Symp. Biocomputing Conf., 2003, vol. 8, pp. 77-88.

[7] Cataldo M., Gaetano R., Marco de G., Pasquale L., Giovanni S. Natural Language Justifications for Recommender Systems Exploiting Text Summarization and Sentiment Analysis . 2019 pp.63-73. http://ceur-ws.org/Vol-2495/paper8.pdf

[8] Or B. and Courtenay C. 2017. Explanation and Justification in Machine Learning: A Survey. In IJCAI-17 Workshop on Explainable AI (XAI), VOL. 8, NO.1. pp 1- 13

[9] Radev, D.R., Jing, H., Sty, M., Tam, D.: Centroid-based summarization of multiple documents. Inf. Process. Manage. 40(6), 919-938 (2004)

[10] Mikolov, T., Sutskever, I., Chen, K., Corrado, G.S., Dean, J.: Distributed representations of words and phrases and their compositionality. In: NIPS. pp. 3111-3119 (2013)

[11] Jianmo N., Jiacheng L., and Julian M.. 2019. Justifying recommendations using distantly-labeled reviews and fine-grained aspects. In Proceedings of the 2019 Conference on Empirical Methods in Natural Language Processing and the 9th International Joint Conference on Natural Language Processing (EMNLP-IJCNLP). pp. 188-197.

[12] Arpit R. and Derek B. 2017. Explanation Chains: Recommendation by Explanation. RecSys '17 Poster Proceedings, Como, Italy, August 27-31, 2017, 2 pages.

[13] Mann, W.C. and Sandra A. T. (1988). "Rhetorical Structure Theory: Toward a functional theory of text organization." Text 8 (3): 243-281.

[14] Jacob D., Ming-Wei C., Kenton L., and Kristina T. 2019. Bert: Pre-training of deep bidirectional transformers for language understanding. In Proceedings of the Conference of the North American Chapter of the Association for Computational Linguistics (NAACL), 2019. pp. 2324-2335.

[15] Yongfeng Z., Guokun L., Min Z., Yi Z., Yiqun L., and Shaoping M. 2014. Explicit factor models for explainable recommendation based on phrase-level sentiment analysis. In Proceedings of the 37th international ACM SIGIR conference on Research \& development in information retrieval , pp. 8392. ACM

[16] Or B., Kathleen M. Generating Justifications of Machine Learning Predictions. 1st International Workshop on Data-to-text 2015 http://www.cs.columbia.edu/ orb/papers/d2t_2015.pdf

[17] Mohamed Hédi Maâloul. Approche hybride pour le résumé automatique de textes. Application à la langue arabe. $\mathrm{PhD}$ thesis, pp 17- 43, 18 décembre 2012. https://tel.archives-ouvertes.fr/tel00756111/file/These.pdf

[18] Mustafa B. and Raymond J. M.. 2005. Explaining recommendations: Satisfaction vs. promotion. In Beyond Personalization Workshop, IUI, Vol. 5. 153. pp 1,pp 7.

[19] Roza Lémdani. Système Hybride d'Adaptation dans les Systèmes de Recommandation. Thèse de Doctorat de 1'Université Paris-Saclay préparée à CentraleSupelec. pp 23- 33. 11 juillet 2016. https://www.theses.fr/2016SACLC050.pdf

[20] Kyelem Y., Kabore K.K., Bassole D. (2022) Hybrid Approach to Cross-Platform Mobile Interface Development for IAAS. In: Shakya S., Bestak R., Palanisamy R., Kamel K.A. (eds) Mobile Computing and Sustainable Informatics. Lecture Notes on Data Engineering and Communications Technologies, vol 68. Springer, Singapore. https://doi.org/10.1007/978-981-16-1866-6_16

(C) 2021 By AIRCC Publishing Corporation. This article is published under the Creative Commons Attribution (CC BY) license. 